\title{
Catalysing the production of multiple arm carbon octopi
}

\section{nanostructures}

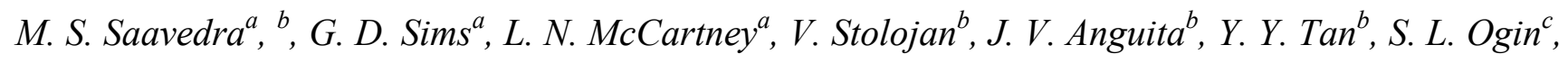
P. A. Smith ${ }^{c}$ and S.R.P. Silva ${ }^{b^{*}}$

${ }^{a}$ National Physical Laboratory, Materials Division, Teddington, TW11 0LW, UK

${ }^{\mathrm{b}}$ Advanced Technology Institute, University of Surrey, Guildford, GU2 7XH, UK

${ }^{\mathrm{c}}$ Division of Mechanical, Medical and Aerospace Engineering, University of Surrey, Guildford, GU2 7XH, UK

* Corresponding author. Tel: $+44(0) 1483$ 689825. Fax: $+44(0) 1483$ 686081. E-mail address: s.silva@surrey.ac.uk (S. R. P. Silva) 


\section{Abstract}

Octopus-like carbon nanofibers with leg diameters as small as $9 \mathrm{~nm}$ are reported, with a high yield over large areas, using a unique photo-thermal chemical vapour deposition system. The branched nature of these nanostructures leads to geometries ideal for increasing the surface area of contacts for many electronic and electrochemical devices. The manufacture of these structures involves a combination of a polyacrylonitrile/polysiloxane film covering the surface of cupronickel catalysts, supported on silicon. Acetylene is used as the carbon feedstock. High-resolution electron microscopy revealed a relationship between the geometry of the nanoparticles and the catalytic growth process, which can be tuned to maximise geometries (and therefore the surface area) and was obtained with a catalyst size of $125 \mathrm{~nm}$. The technique proposed for growing these carbon octopi nanostructures is ideal to facilitate a new insitu transfer film process to place high-density carbon structures on secondary surfaces to produce low resistance and high capacitance all-carbon contacts.

\section{Introduction}

The continued drive for miniaturisation of integrated circuits demands that the constituent electronic components follow a similar trend, for example, in providing lightweight contacts with larger surface areas. Conductive, carbon nanostructures have been promoted as a route to achieving such lightweight large surface area materials [1]. It is suggested that the octopus-like nanostructures are an ideal solution for fulfilling these requirements, owing to their low-density and large surface area. Octopi-carbon nanofibers, consisting of a central catalyst from which carbon nano-fibre legs radiate, have been described previously in the literature [2-10], but to date their production has been at high temperatures, incompatible with back-end-of-line semiconductor device processing. Octopi structures can offer an advantage over conventional carbon nanotube/nanofibre forests in that the multiple carbon "legs" are free of bundles and thus offer greater surface area to volume ratios and higher diffusion speeds for the 
adsorption of larger molecules, which is useful for supercapacitor contacts. Stacking multiple layers of these nanostructures would produce even larger surface areas that are ideal for applications such as contacts for organic photovoltaics (OPV) and organic light emitting diodes (OLED).

One of the most studied routes for the production of specific "designer" nano-materials has been the use of nano-catalysts to nucleate designed carbon structures. Methods of growth involve varying the catalyst and gaseous carbon feedstock elements and quantities, where the catalysts and gases used often consist of multiple combinations of materials. The unusual aspect of this study is the role a polyacrylonitrile (PAN) and polysiloxane based polymer film plays on the surface of the catalysts during the growth of the octopi structures. The nucleation of the octopi structure is also found to be critically dependent on the copper content in the nickel catalyst. The role of catalysts in the formation of octopuslike nanostructures has been investigated using copper (in metal catalysts ranging between 3\% and $80 \%$ $\mathrm{Cu}$ ), but the exact growth mechanism and the role of the catalyst has still to be understood [2 - 10]. This work also presents fundamental studies on the experimental conditions that give rise to octopistructures, as well as statistics on octopus leg numbers, diameters and lengths which enable the proposal of a simple growth mechanism based on two well-known processes occurring during the annealing of a copper and nickel catalyst system: the Kirkendall effect [11, 12] and Ostwald ripening [13].

Typically, the growth of octopus nanofibers using conventional thermal CVD processes and methane gas, involves relatively high temperatures up to $950{ }^{\circ} \mathrm{C}[3-11]$. This limits the process to hightemperature resistant substrates. In the method used in this work, which is the photo-thermal CVD (PTCVD) method, energy is coupled directly into the catalyst while the substrate is water-cooled, allowing for the use of flexible plastic substrates, often required in large area plastic electronics [14, 15]. Acetylene has been used as the carbon feedstock owing to its lower cracking temperature relative to methane, enabling PTCVD thermal processing at lower temperatures. 


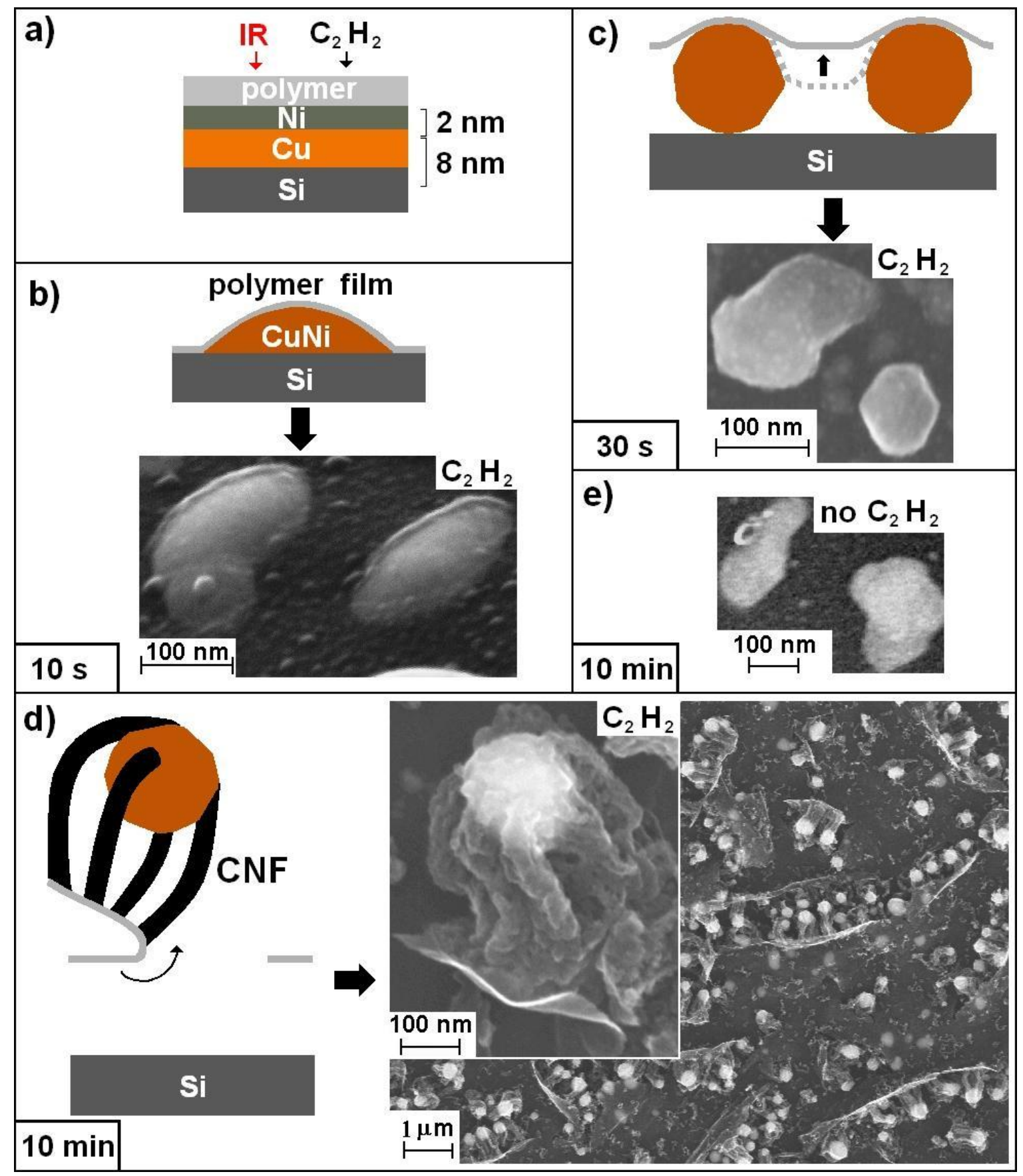

Figure 1 Schematics showing a) the initial structure introduced in the PTCVD growth system and b)-d) the stages of growth of the octopi with the corresponding SEM micrographs; b) shows a catalyst island 10 seconds after introducing acetylene - the islands formed from the metal bi-layer within the 3 minutes of annealing prior to introducing acetylene and may have increased in size under the film over time; c) shows the catalyst 30 seconds after the introduction of acetylene, when more faceted catalysts have formed and the film was observed to have risen from the wafer; $d$ ) shows catalyst islands 10 minutes after introducing acetylene where the film split into multi-directional slits from which octopus structures 
could be observed as pea-pod like structures. The octopus legs were attached to the polymer film, so that the catalysts appear to be moving away from the film, suggesting tip growth at the surface of the film; e) shows the result when acetylene was not introduced where the film remained intact and the islands remained the same as they were 10 seconds into the growth.

\section{Experimental}

The samples were prepared on silicon substrates, typically of dimension $2 \mathrm{~cm} \times 7 \mathrm{~cm}$, that were cleaned and then coated with the bilayer catalyst. Two different bilayer configurations have been used. In samples labelled "1", the metal coating had $2 \mathrm{~nm}$ of $\mathrm{Ni}$ sputtered on top of $8 \mathrm{~nm}$ of $\mathrm{Cu}$ (Figure 1), while samples labelled " 2 " had the two layers of sputtered metal deposited in the reverse order. The first type of sample is expected to catalyse only carbon nanotubes if the surface remains pure nickel [16]. However, as the copper has a lower melting point than nickel it may diffuse into the top nickel layer producing a cupronickel alloy, which can lead to herringbone-like carbon nanofiber (CNF) growth [17]. The second type of sample was produced to test the extent of mixing between copper and nickel on the surface of the catalyst. If the annealing time is too short and the thickness of the different metals relatively thick, the $\mathrm{Cu}$ and $\mathrm{Ni}$ will not mix, giving different nanostructures to the observed octopus nanofibers. The sputtering was carried out using a magnetron sputter system (MPS 500, JLS Designs Ltd). A copper tape (Agar Scientific, G3940) was used to cover one half of the metal coated samples so that the polymer surface of the tape (having a high concentration of PAN and polysiloxanes) adhered to the surface of the metal bi-layer. The growth of the octopi structures was carried out in a PTCVD chamber (Surrey NanoSystems 1000n $[14,15]$ ) at an average substrate surface temperature of $511{ }^{\circ} \mathrm{C}$, and a bulk substrate temperature of $392{ }^{\circ} \mathrm{C}$ monitored by a thermocouple and a pyrometer, respectivley. Hydrogen was introduced into the chamber at $100 \mathrm{sccm}$ (standard cubic centimeters per minute) throughout heating for 10 minutes to reduce any metal oxides. When the correct temperature was reached, acetylene $\left(\mathrm{C}_{2} \mathrm{H}_{2}\right)$ was introduced at $6 \mathrm{sccm}$, maintaining a constant pressure of 2 Torr. The growth process for different specimens was stopped 10 seconds, 30 seconds and 10 minutes after 
acetylene was introduced, in order to investigate the growth mechanism at different stages. An additional run carried out for 10 minutes in the absence of acetylene demonstrated that the polymer film alone was not the carbon feedstock, as no octopi were found.

\section{Results and Discussions}

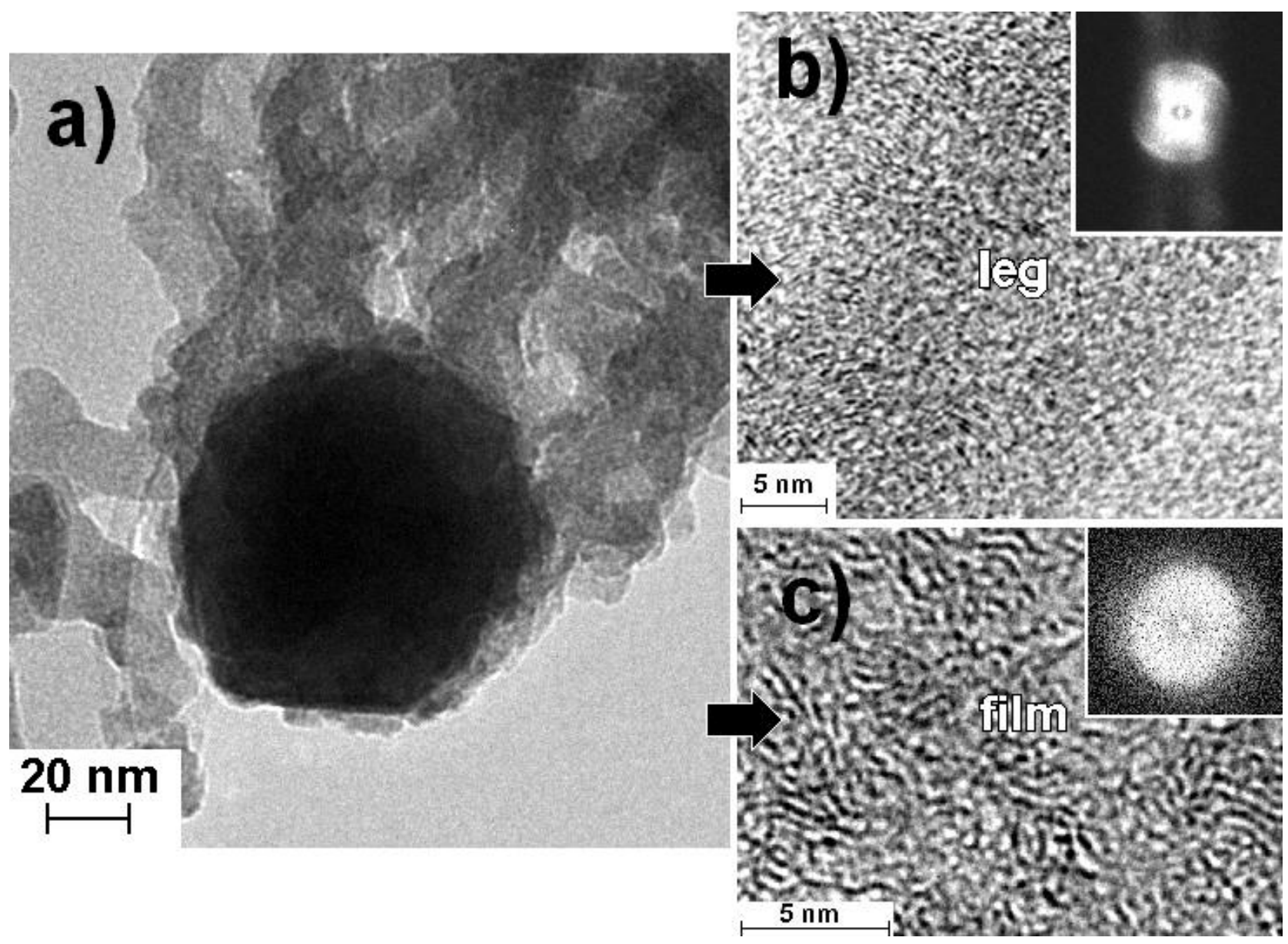

Figure 2 HR-TEM micrograph of a) an octopus nanostructure showing non-hollow carbon legs that grew from a faceted catalyst; b) the atomic structure of a single octopus leg a defective graphitic structure, with average lattice spacing $0.34 \mathrm{~nm}$, as calculated from the inset fast Fourier transform (FFT), c) the graphitic film showing short-range crystallinity with lattice fringe spacings of $0.36 \mathrm{~nm}$ as determined from the inset FFT.

Octopus-like nanostructures completely covered the whole substrate that was under the copper tape, an area equal to several centimetres squared in size. The octopus growth only occurred on the samples 
where the copper was deposited prior to the nickel. For the case where nickel was deposited prior to the copper, a single fibre grew from each catalyst. Trial samples prepared with only nickel, having the same total film thickness, did not show any octopus-like growth. The literature suggests that herringbone-like structures should be expected from a copper-nickel alloy catalyst [17]; however, this structure is only seen in the areas not covered by the polymer. In order to identify the reasons for this, the growth mechanism of the fibers was investigated and the stages of growth are shown schematically in Figure 1, where the times indicate elapsed time after introducing the acetylene. During the 3 minute ramping to temperature time prior to the introduction of acetylene into the chamber, the metal bilayer was found to have formed catalyst islands below the polymer film, as was expected [18]. After 10 seconds, the catalyst appear as islands below the polymer layer (Figure 1b). After 30 seconds growth, the catalysts evolved into faceted spheres (Figure 1c), a process that did not occur in the absence of acetylene (Figure 1e). This indicates that acetylene has permeated through the $7 \mathrm{~nm}$ thick polymer film (measured using a scanning electron microscope, SEM) to reach the catalyst. After 10 minutes of growth, the octopi were observed in slits that have formed in the polymer film $(\sim 5 \mathrm{~nm}$ thick $)$, or bursting out from beneath it (as shown in Figure 1d). The slits were not observed in the absence of acetylene, indicating that the slit formation is a consequence of octopus leg growth (Figure 1e). It was found that the polymer film graphitised (Figure 2) and that the octopus legs were attached to the film and not to the silicon substrate, indicating a tip-growth mechanism, where the film, and not the silicon, acted as the substrate (Figure 1d). This growth mechanism would therefore be suitable for an in-situ transfer process for manufacturing a graphitised polymer film with attached carbon octopi nanostructures, which can be removed to make an all-carbon contact.

The different growth stages can be described in terms of the polymer film. The graphitised carbon film is a residue from the polymer that melted, decomposed and evaporated at temperatures above $200{ }^{\circ} \mathrm{C}$, coating the catalyst tightly as shown in Figure 1b. During the graphitisation, the polymer film lost a fraction of its mass and shrank in the process. The shrinking of the film caused it to rise and flatten 
between catalysts, as shown in Figure 1c. The gases forming as a consequence of the polymer film decomposition at $300{ }^{\circ} \mathrm{C}$ (mainly $\mathrm{C}_{2} \mathrm{~N}_{2}, \mathrm{NH}_{3}, \mathrm{HCN}, \mathrm{C}_{4} \mathrm{H}_{5} \mathrm{~N}, \mathrm{CH}_{3} \mathrm{CN}, \mathrm{C}_{3} \mathrm{H}_{3} \mathrm{~N}$ and $\mathrm{H}_{2} \mathrm{O}[19,20]$ ) may have helped the film to rise. At the same time, the catalysts change from flat, wide islands to faceted spheres. The catalyst shape-change (and the CNF growth) occurred only in the presence of acetylene. The shape change increased the height of the catalysts (while the volume was conserved), also contributing, no doubt, to raising the graphitised polymer film. Eventually, the combination of tension due to the shrinking of the film, the gas pressure due to the film decomposition, and the local irregularities caused by the growing octopi nanostructures, lead to splitting of the film, revealing the octopi in pea-pod-like structures (Figure 1d).

Further characterisation aided the understanding of the structure and its growth. A high-resolution scanning transmission electron micrograph (HR-STEM) of an octopus CNF and a typical leg are shown in Figure 2a and b, respectively. Owing to the higher resolution of the STEM images, it was possible to distinguish the legs aiding their counting. As the octopi coagulated in the solution prepared for STEM imaging, it was not possible to determine the octopus leg length. STEM images confirmed the irregular polygon structure of the catalysts (Figure 2a), indicating irregularly sized facets. The octopus legs appear to grow on the facets, the vertices and edges between the facets. The absence of visible walls in the legs (Figure 2b) indicates that the octopus-legs are not hollow. The atomic structure of the legs is graphitic (Figure 2b), with arched atomic lattice fringes occurring owing to the wavy nature of the legs. The lattice fringe spacings of $0.34 \pm 0.04 \mathrm{~nm}$ matched those of graphite at $0.34 \mathrm{~nm}$ [21]. Energy dispersive X-ray analysis (EDX) confirmed the carbon composition of the legs and the film to which they were attached. In addition, EDX and electron energy loss spectroscopy (EELS) confirmed the prevalence of copper on the surface of the catalyst with copper and nickel percentages of $83 \%$ and $17 \%$ on the surface and $73 \%$ and $27 \%$ near the centre, respectively. This is expected, as copper is known to segregate at about $500{ }^{\circ} \mathrm{C}$ [7] from the copper-nickel mixture arising from inter-diffusion and during the cooling of the catalyst. The segregation of copper when heated is as consequence of the Kirkendall effect [11]. The 
increase in size of some catalysts at the expense of others is likely to be an Ostwald ripening process. In order to lower the energy of the system, the unstable copper and nickel atoms will migrate from the smaller particles to the larger particles. An approximately bimodal distribution was obtained for the octopus catalysts, where modes 1 and 2 have a mean catalyst diameter of $\sim 122 \mathrm{~nm}$ and $\sim 222 \mathrm{~nm}$ respectively, as shown in Figure 3a. The two distributions may be indicative of the effect of the Ostwald ripening process.
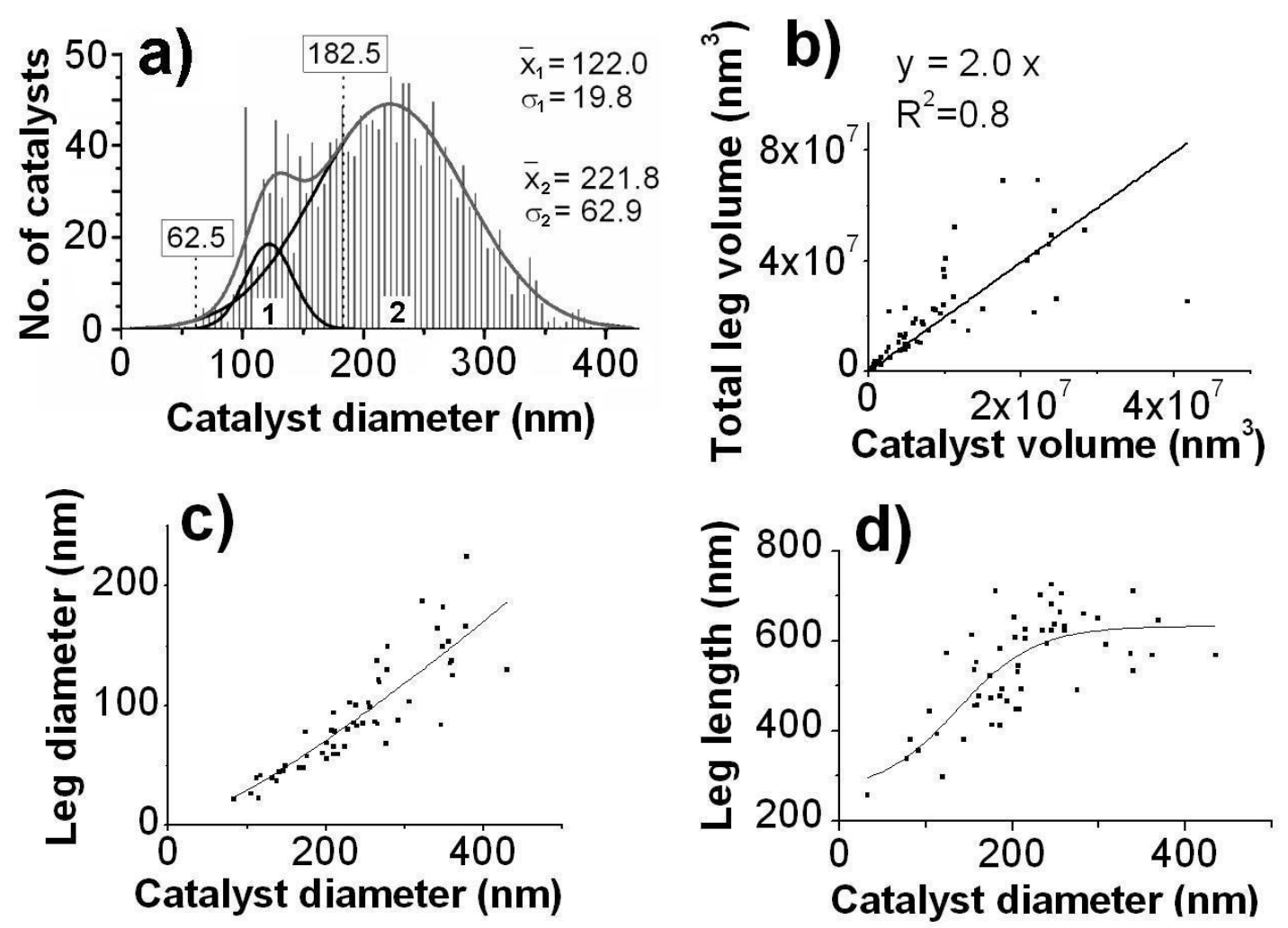

Figure 3 SEM measurements gathered for multiple octopi showing a) the bimodal catalyst size distribution for 1275 individual octopi (and the points at which the contribution from population 1 starts and ends, i.e. at $62.5 \mathrm{~nm}$ and $182.5 \mathrm{~nm}$ respectively); b) the total leg volume obtained for 54 octopi by multiplying SEM measurements of c) the average leg diameter per catalyst and d) the average leg length.

The two peaks might result from the smaller catalysts becoming smaller and the larger catalysts increasing in size as a result of Ostwald ripening. The PAN and polysiloxane based polymer film 
increases the ripening time by slowing down the rate at which the acetylene diffuses to the catalyst islands.

The octopus leg diameter (Figure 3c), leg length (Figure 3d) and number of legs for a given catalyst size range (Figure 3a) were used to produce a plot of the leg volume as a function of catalyst volume. It was found that, on average, the total carbon leg volume catalysed increased approximately linearly with the increasing catalyst volume and is twice as large as the catalyst volume (Figure $3 \mathrm{~b}$ ) where the legs were modelled to be perfect cylinders. On the same plot, an interesting feature is the apparently linear increase in the scatter associated with the total leg volume as the catalyst volume increased. This means that the larger the catalyst, the larger the space that is available for the formation of larger diameter fibers, making it more likely to find a larger range of differently sized legs. The scatter should be minimised in order to obtain a better control over the increase in surface area desired. This can be achieved by considering only the catalysts in the $<200 \mathrm{~nm}$ regime. STEM images were also used to determine the dependence of the number of legs on the diameter of the catalyst (Figure 4). Focusing on diameters $<200 \mathrm{~nm}$, it can be observed that at the catalyst diameter for which catalyst population 1 in Figure $3 \mathrm{a}$ is highest, which occurs at about $125 \mathrm{~nm}$, the number of legs is at its maximum and the diameter of the legs is close to the minimum value.

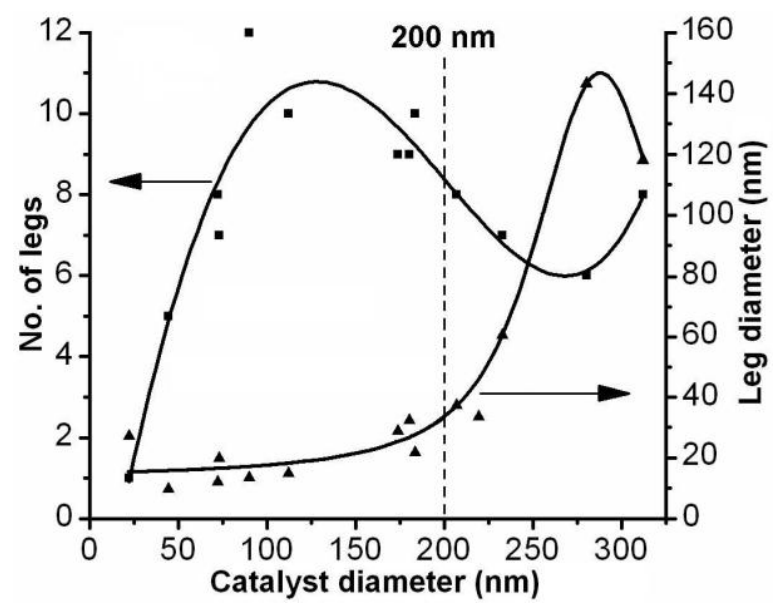

Figure 4 Catalyst and average leg diameters for 13 octopi determined using a STEM. 
In conventional carbon nanotube/fibre growth, the number of fibers catalysed remains approximately the same up to a catalyst size of $300 \mathrm{~nm}$, after which their number increases linearly with increasing catalyst diameter [22]. Also, the average diameter of conventional nanofibers increases linearly with increasing catalyst diameter between $\sim 30$ and $460 \mathrm{~nm}$, with a gradient of approximately $\sim 1.0$ (no units) [23]. In the octopus system described here, the number of legs/catalyst diameter relationship is different as the number of legs increases relatively linearly with increasing catalyst diameter up to a catalyst diameter of $\sim 100 \mathrm{~nm}$, with a steeper gradient than previously reported [22] (Figure 4). This unexpected behaviour might suggest a near-conventional growth process for a catalyst $\sim 25-100 \mathrm{~nm}$ and a reversed trend at catalyst sizes between $100 \mathrm{~nm}$ and $250 \mathrm{~nm}$. The more complex growth of larger catalysts could be attributed to nearing bulk sizes, and thus displaying more complex bulk behaviour.

The conventional growth process of carbon nanofibers indicates the surface diffusion of carbon and subsequent crystallisation into fibers, as well as preferential step-edge carbon feedstock dissociative adsorption and nucleation [24 - 26]. There has also been evidence of bulk diffusion by considering the effects of nickel crystal orientations [27]. In the case of the octopus system, the nucleation process could occur on the surface at step edges and defects formed by the segregation of copper, which might explain why the octopi only grow in a copper nickel-alloy system. Surface diffusion can be determined by analysing the amount of catalysed carbon as a function of the catalyst size, so that for an increasing catalyst surface area, the catalysed carbon should increase linearly. The same can be method can be used for bulk diffusion and for this reason it is often possible to determine a surface or bulk diffusion process by plotting the number of legs against catalyst size. However, neither surface nor bulk diffusion was determined in this case due to the high scatter.

\section{Summary}

The radial growth of thinner, longer, more numerous legged octopi for higher surface areas can be controlled by the size of the catalysts on which they are grown. It is suggested that the branched 
structure of the octopus legs results from the difference in the melt temperatures of copper and nickel; the surface nickel can diffuse into the copper as a consequence of the Kirkendall effect, leaving areas of nickel around the catalyst able to catalyse the carbon. It was found that the diameter and length of the nanofibers depend on the catalyst diameter, which can be tuned by controlling the annealing time of the catalyst, which in turn controls the Ostwald ripening time. It was found that the larger the catalyst, the more likely it is to find a larger range of differently sized legs. Consequently, better control over the surface area can be obtained at small catalyst sizes $<200 \mathrm{~nm}$. It was also found that at a critical catalyst size of $125 \mathrm{~nm}$ the surface area that can be obtained from the octopus geometry was ideal, having a large number of octopus legs. As the polymer film is graphitised during the production of the octopi nanostructures, and the fibers grow preferentially on the graphite, this process could be used for growing nanostructures directly onto graphite / graphene to produce an all-carbon contact with a high surface area.

\section{Acknowledgements}

The work was carried out as part of an Engineering Doctorate Programme in Micro- and NanoMaterials and Technologies, financially supported by the EPSRC, the University of Surrey and the National Physical Laboratory. The authors would like to thank Dr Felicia Green (NPL) and Steven King (NPL) for access to the ToF SIMS equipment and consultation.

\section{References}


[1] Wallace GG, Chen J, Li D, Moulton SE, Razal JM. J. Nanostructured carbon eletrodes. Mater. Chem. 2010;20(18):3553-3562.

[2] Saito Y. Nanoparticles and filled nanocapsules. Carbon 1995;33(7):979-988.

[3] Ermakova MA, Ermakov DY, Chuvilin AL, Kuvshinov GG. Decomposition of methane over iron catalysts at the range of moderate temperatures: the influence of structure of the catalytic systems and the reaction conditions on the yield of carbon and morphology of carbon filaments. J. Catal. 2001;201(2):183-197.

[4] Alstrup I. J. Catal. A new model explaining carbon filament growth and Ni-Cu alloy catalysts. 1988;109(2):241-251.

[5] Bernardo CA, Alstrup I, Rostrup-Nielsen JR. Carbon deposition and methane steam reforming on silica-supported Ni-Cu catalysts. J. Catal. 1985;96(2):517-534.

[6] Tavares MT, Bernardo CA, Alstrup I, Rostrup-Nielsen JR. Reactivity of carbon deposited on nickelcopper alloy catalysts from the decomposition of methane. J. Catal. 198; 100(2):545-548.

[7] Avdeeva LB, Goncharova OV, Kochubey DI, Zaikovskii VI, Plyasova LM, Novgorodov BN et al. Coprecipitated Ni-alumina and Ni-Cu-alumina catalysts of methane decomposition and carbon deposition. II. Evolution of the catalysts in reaction. Appl. Catal. A-Gen. 1996;141(1-2):117-129.

[8] Pradhan D, Sharon M, Kumar M, Ando YJ. Nanosci. Nano-octopus: a new form of branching carbon nanofiber. Nanotech. 2003;3(3):215-217.

[9] Pham-Huu C, Vieira R, Louis B, Carvalho A, Amadou J, Dintzer T et al. About the octopus-like growth mechanism of carbon nanofibers over graphite supported nickel catalyst. J. Catal. 2006;240(2):194-202. 
[10] Li Y, Chen J, Ma Y, Zhao J, Quin Y, Chang L. Formation of bamboo-like nanocarbon and evidence for the quasi-liquid state of nanosized metal particles at moderate temperatures. Chem. Comm. 1999;3(12):1141-1142.

[11] Abbaschian R, Abbaschian L, Reed-Hill RE, Physical metallurgy principles: Stamford CT: Cengage Learning. 2009: 355-357.

[12] Zhao N, Kang J, Li J, Nash P. In situ synthesis of carbon nanostructures supported on metal powders by chemical vapor deposition, The Seventeenth Proceedings of the Annual International Conference on Composites/Nano Engineering (ICCE - 17) ICCE, Hawaii USA, 2009; July p. $26-31$.

[13] Aruga T, Kishi K, Nishijima M. Diffusion and coalescence of bilayer surface-alloy islands of Pd(100)-p(2x2)-p4g-Al. Surf. Sci., 2001;493(1-3):325-330.

[14] Chen GY, Stolojan V, Silva SRP. Top-down heating for low substrate temperature synthesis of carbon nanotubes. J. Nanosci. and Nanotechnol. 2010;10(6):3952-3958.

[15] Shang NG, Tan YY, Stolojan V, Papakonstantinou P, Silva SRP. High-rate low-temperature growth of vertically aligned carbon nanotubes. Nanotechnology 2010;21(50):505604.

[16] Oncel C, Yurum Y. Carbon nanotube synthesis via the catalytic CVD method: a review on the effect of reaction parameters. Fuller. Nanotub. Car. N. 2006;14(1):17-37.

[17] Yoon S-H, Lim S, Hong S-H, Qiao W, Whitehurst DD, Mochida I et al. A conceptual model for the structure of catalytically grown carbon nano-fibers. Carbon 2005;43(9)1828-1838.

[18] Carey JD, Ong LL, Silva SRP. Formation of low-temperature self-organized nanoscale nickel metal islands. Nanotechnology 2003;14(11)1223-1227.

[19] Wilkie CA, Xue TJ, Mckinney MA. The thermal degradation of polyacrylonitrile. Polym. Degrad. Stabil. 1997;58(1-2):193-202. 
[20] Monahan AR. Thermal degradation of polyacrylonitrile in the temperature range $280-450{ }^{\circ} \mathrm{C} . \mathrm{J}$. Polym. Sci. Pol. Chem. 1966;4(10):2391-2399.

[21] Guan L, Suenaga K, Iijima S. Smallest carbon nanotube assigned with atomic resolution accuracy. Nano Lett. 2008;8(2):459-462.

[22] Teo KBK, Lee S-B, Chhowalla M, Semet V, Binh VT, Groening O et al. Plasma enhanced chemical vapour deposition carbon nanotubes/nanofibres - how uniform do they grow?. Nanotechnology $2003 ; 14(2): 204-211$.

[23] Chhowalla M, Teo KBK, Ducati C, Rupesinghe NL, Amaratunga GAJ, Ferrari AC et al. Growth process conditions of vertically aligned carbon nanotubes using plasma enhanced chemical vapor deposition. Appl. Phys 2001;90(10):5308-5317.

[24] Chen GY, Jensen B, Stolojan V, Silva SRP. Growth of carbon nanotubes at temperatures compatible with integrated circuit technologies. Carbon 2011;49(1):280-285.

[25] Helveg S, López-Cartes C, Sehested J, Hansen PL, Clausen BS, Rostrup-Nielsen JR et al. Atomicscale imaging of carbon nanofibre growth. Nature 2004;427:426-429.

[26] Stolojan V, Tison Y, Chen GY, Silva R. Controlled growth-reversal of catalytic carbon nanotubes under electron-beam irradiation. Nano Lett. 2006;6(9):1837-1841.

[27] Sharma R, Iqbal Z. In situ observations of carbon nanotube formation using environmental transmission electron microscopy. Appl. Phys. Lett. 2004;84(6):990. 